Meta

Journal des traducteurs

Translators' Journal

\title{
La traduction de la terminologie philosophique
}

\section{Siobhan Brownlie}

Volume 47, numéro 3, septembre 2002

URI : https://id.erudit.org/iderudit/008017ar

DOI : https://doi.org/10.7202/008017ar

Aller au sommaire du numéro

Éditeur(s)

Les Presses de l'Université de Montréal

ISSN

0026-0452 (imprimé)

1492-1421 (numérique)

Découvrir la revue

Citer cet article

Brownlie, S. (2002). La traduction de la terminologie philosophique. Meta, 47(3), 296-310. https://doi.org/10.7202/008017ar

\section{Résumé de l'article}

Après avoir examiné certaines caractéristiques générales de la terminologie philosophique qui sont importantes pour la traduction des termes, l'auteur aborde la traduction du lexique philosophique dans un corpus de textes, mettant en évidence la différence entre le traitement des termes techniques et celui du lexique général. L'examen plus détaillé du corpus permet de complexifier cette perspective binaire. L'auteur s'attache ensuite à trouver l'explication des pratiques traductionnelles dégagées, ce qui nécessite d'explorer le contexte social où circulent les termes traduits.
Ce document est protégé par la loi sur le droit d'auteur. L'utilisation des services d'Érudit (y compris la reproduction) est assujettie à sa politique d'utilisation que vous pouvez consulter en ligne.

https://apropos.erudit.org/fr/usagers/politique-dutilisation/ 


\title{
La traduction de la terminologie philosophique
}

\author{
SIOBHAN BROWNLIE \\ Université de Queensland, Brisbane, Australie
}

\begin{abstract}
RÉSUMÉ
Après avoir examiné certaines caractéristiques générales de la terminologie philosophique qui sont importantes pour la traduction des termes, l'auteur aborde la traduction du lexique philosophique dans un corpus de textes, mettant en évidence la différence entre le traitement des termes techniques et celui du lexique général. L'examen plus détaillé du corpus permet de complexifier cette perspective binaire. L'auteur s'attache ensuite à trouver l'explication des pratiques traductionnelles dégagées, ce qui nécessite d'explorer le contexte social où circulent les termes traduits.
\end{abstract}

\begin{abstract}
After examining certain general characteristics of philosophical terminology which are important for the translation of the terms, the author studies the translation of philosophical lexis in a corpus of texts, highlighting the difference in treatment of technical terms and general lexis. A more detailed study of the corpus reveals complexities beyond that binary dichotomy. The author then aims to produce explanations for the translational practices which have been adduced. This necessitates exploring the social context in which the translated terms circulate.
\end{abstract}

\section{MOTS-CLÉS/KEYWORDS}

contexte social, lexique général, procédés de traduction, termes techniques, terminologie philosophique

\section{PRÉSENTATION}

D’après Alain Rey les subtiles frontières des termes-concepts définissent le propre de la rhétorique philosophique, car sans l'architecture lexicale le discours ne serait plus philosophique, mais littéraire ou poétique (Rey 1990: 781). Il s'agit là d'un critère interne pour affirmer le caractère philosophique d'une production textuelle. Si les termes constituent un élément crucial des textes philosophiques, il importe d'étudier le sort que connaissent ces termes dans les traductions des textes.

La présente étude ne traite pas la question de façon globale. Elle s'appuie plutôt sur un corpus qui comprend neuf articles et deux livres d'un philosophe français contemporain, Jean-François Lyotard (1925-1998), et les traductions de ces textes en langue anglaise. Pour quatre textes ou extraits de textes il existe deux traductions qui sont incluses dans le corpus; cela permet de comparer les procédés parfois divergents de deux traducteurs devant la même tâche de traduction. Les textes du corpus ont été publiés principalement aux États-Unis et en Angleterre, entre 1978 et 1993. Les traducteurs des textes sont pour la plupart des universitaires anglophones (américains, anglais ou australiens), et les lecteurs des textes se trouvent aussi dans les milieux universitaires anglophones (professeurs et étudiants). C'est grâce aux textes traduits que les universitaires anglophones ont connu l'œuvre de Lyotard, qui a influencé des 
débats en théorie littéraire et en philosophie, surtout concernant la question du postmoderne.

Bien que notre étude soit basée sur un seul corpus, il semble que les caractéristiques de la traduction de la terminologie que nous relevons soient partagées par d'autres traductions d'œuvres philosophiques. En témoigne la traduction de citations que l'on trouve dans les textes de Lyotard, ainsi que les commentaires de chercheurs que nous citerons au cours de l'exposé. D'autres études attirent cependant l'attention sur des traits variables en matière de traduction philosophique, qui tiennent d'une part au couple de langues en présence (par exemple le couple allemand-russe, voir Petioky 1994) et d'autre part à l'approche idiosyncrasique de certains traducteurs (par exemple, Frederico Mueller, traducteur brésilien de Rudolf Steiner, voir RothNeves 2000). Avant de passer à l'examen de notre corpus, nous considérerons quelques caractéristiques de la terminologie philosophique.

\section{LA TERMINOLOGIE PHILOSOPHIQUE}

Comme d'autres activités spécialisées, la philosophie possède ses termes techniques. Les termes dénotent des concepts renvoyant à un ensemble de préoccupations dites philosophiques, et ils constituent un outil facilitant la communication entre spécialistes. À la différence cependant de la terminologie technique et scientifique où les termes sont élaborés et normés au sein d'une communauté de spécialistes, les termes philosophiques sont associés de façon intime à des individus. En effet l'individualité de la parole, qui est pourtant fondée en socialité, s'affirme en attribuant des valeurs données à certaines formes - mots et expressions: c'est l'œuvre d'élaboration terminologique des créateurs (Rey 1990: 781). Pour Michèle Lamont la création d'un ensemble de termes distinctifs est un critère essentiel à la légitimation intellectuelle d'un philosophe, qui consiste à attribuer à l'œuvre du philosophe un certain statut dans les milieux intellectuels. L'ensemble de termes distinctifs constitue une «marque théorique» qui permet d'abord de distinguer et de reconnaître l'œuvre du philosophe, et qui favorise ensuite la rapide dissémination et l'assimilation de la théorie grâce au rôle des termes distinctifs en tant qu' «étiquettes» (Lamont 1987: 592). Après leur création, donc, les termes accèdent à l'ustensilité sociale; ils sont repris et travaillés par les commentateurs, les herméneutes, les didacticiens et les lexicographes (Rey 1990: 781).

Le terme philosophique est souvent un mot existant auquel le philosophe donne un nouveau sens ou un sens plus spécifique. Il s'agit là d'un emprunt fait à un autre 'domaine'. C'est justement en raison de l'emprunt de termes que dans des travaux récents sur la terminologie on propose de substituer au concept de 'domaine' celui de 'réseau de nœuds'. Inspiré des écrits de Michel Serres, ce concept a l'avantage de nier l'étanchéité que comporte la notion de domaine, et de mieux décrire la réalité du fonctionnement des disciplines, c'est-à-dire l'importance des contacts interdisciplinaires. Les mots, et les schémas de pensée circulent (Gaudin 1995: 231). Il en va de même des relations entre ce que l'on a coutume d'appeler la langue générale et les langues de spécialité. Il est à noter, toutefois, que l'ouverture sur d'autres disciplines ou sur d'autres genres de langage ne contredit pas l'existence de particularismes, car la circulation donne lieu à l'appropriation et à la transformation de sens aux jonctions des réseaux. Il existe en outre certains termes inventés par les spécialistes d'une 
discipline (néologismes), et peu employés en dehors de la discipline. Dans le cas de la philosophie on trouve selon Rey deux types de termes: des termes empruntés aux vocabulaires courants (par exemple 'être' ou 'néant'), et des termes qu'on ne trouve pas dans les vocabulaires courants (par exemple 'être-là') (Rey 1990 : 779). Les termes de Lyotard appartiennent à la première catégorie; ils proviennent de la langue générale (par exemple 'enchaînement') ou d'un lexique spécialisé tel que le lexique juridique (par exemple 'dommage', 'litige'). Il convient de noter que dans un certain nombre de cas les termes techniques de Lyotard ne sont pas des mots pris individuellement mais des syntagmes (par exemple 'grand récit', 'régime de phrase'). Lyotard attribue des sens spécifiques à ces termes qui dénotent des concepts centraux de sa philosophie. À différents moments dans l'évolution de sa pensée, des ensembles de termes constituent des réseaux où un terme est défini par ses rapports aux autres termes de l'ensemble. Voici à titre d'indication le début de l'énoncé définitionnel du terme 'différend': "À la différence d'un litige, un différend serait un cas de...» (Lyotard 1983: 9, nous avons mis en italique les termes techniques).

L'emploi du terme 'différend' dans les textes de Lyotard illustre un aspect important mais parfois négligé de la terminologie, l'évolution des concepts et des termes. Cette évolution comporte un flou et un flux terminologiques, des mises au point sémantiques, et des moments de stabilisation. Se pencher sur la question de l'évolution des termes nécessite une étude diachronique des textes. Lyotard a présenté le texte 'Judicieux dans le différend' lors d'un colloque tenu en 1982 (les actes du colloque ont paru par la suite en 1985 (Lyotard 1985)). Dans ce texte Lyotard parle d'un «différend illusoire» et d'un «différend véritable». Un «différend illusoire» peut être dissipé, alors que dans un « différend véritable » il s'agit d'une différence irréductible entre les deux parties, en somme de leur incommensurabilité (Lyotard 1985: 209-213). C'est la deuxième définition que l'auteur adoptera de façon explicite comme sens du terme 'différend' dans le livre Le différend, publié en 1983 (Lyotard 1983). On s'aperçoit dans cet exemple d'un processus de technicalisation du terme sur lequel nous reviendrons plus loin, car les conséquences peuvent être importantes pour l'activité de traduction des textes.

\section{TRADUIRE LE LEXIQUE PHILOSOPHIQUE: DISTINCTION ENTRE LES TERMES TECHNIQUES ET LE LEXIQUE GÉNÉRAL}

Dans un texte philosophique la terminologie technique revêt une très grande importance qualitative, même si, quantitativement, la langue générale est prépondérante. Puisque les termes philosophiques sont élaborés par des individus dans un contexte culturel particulier et dans une langue spécifique, il n'en existe pas, par contraste avec les termes technologiques et scientifiques, d'équivalents préexistants dans une autre langue, du fait de l'élaboration simultanée de concepts et d'activités dans le domaine en question. Ce sont les traducteurs du philosophe qui ont donc la responsabilité de créer des équivalents.

En ce qui concerne la traduction du lexique, le trait le plus saisissant de notre corpus est la différence entre le traitement des mots considérés comme termes techniques ou mots-clés ${ }^{1}$, et celui des mots considérés comme faisant partie intégrante du lexique général. D’autres chercheurs ont également remarqué cette distinction 
dans les procédés traductionnels pour le lexique d'un texte philosophique. Ainsi Henri Meschonnic à propos des traductions françaises d'un texte de Humboldt, Ueber die Aufgabe des Geschichtschreibers:

Le texte est respecté dans sa rigueur, mais cette rigueur est seulement substantive, conceptuelle. Par quoi paraît une certaine idée du texte philosophique: hors la technicité - qui suppose une confusion entre le concept et le mot — tout le reste est littérature... à l'intérieur de l'exactitude relative observée par la stabilité de la concordance lexicale pour les termes principaux... apparaît un traitement du mot comme sens-dans-soncontexte, qui lui enlève sa mémoire de texte. (Meschonnic 1985: 219, 221)

Meschonnic signale donc qu'en traduisant les termes techniques ou les termes principaux de Humboldt, le traducteur reproduit la systématique du texte-source par l'uniformité de ses choix — il traduit toujours un terme de la même façon — et par la reproduction d'ensembles de mots partageant la même racine. Quant à la traduction des mots qui ne sont pas considérés comme des termes principaux, le traducteur emploie une diversité de traductions pour le même mot qui apparaît plusieurs fois dans le texte, et ne reproduit pas d'autres liens cohésifs du tissu lexical. Meschonnic proteste contre ce deuxième élément de la double pratique, le traitement du lexique non-technique, car pour lui le «mode de signifier» fait partie du sens; par la nonprise du texte comme système, le «rythme du sens» est atteint (Meschonnic 1985: 221).

J.-R. Ladmiral en revanche semble soutenir la double pratique, quoique ses propos soient nuancés car il insiste sur la nécessité de choisir parmi des procédés potentiels en fonction de la spécificité de chaque cas. Pour Ladmiral le traducteur doit décider si un élément du texte ressortit à la parole de l'auteur ou seulement à la langue-source dont il se sert. Dans le premier cas il faudra «faire passer» l'information; dans le second cas, on pourra se contenter de mettre en ouvre les ressources propres à la langue-cible. En ce qui concerne les mots et les expressions de l'auteur il s'agit de les considérer comme des termes-clés et de procéder à la «terminologisation traductive» de ces termes. La terminologisation consiste à produire des termes-cible que l'on utilisera chaque fois que le terme apparaît, le terme-cible étant soit une traduction littérale du terme-source, soit un mot apparenté, comportant en tout cas un usage néologique de par le sens et parfois de par la forme de la langue-cible. La prise en compte de traductions antérieures choisies par d'autres traducteurs du philosophe est un autre aspect important de la terminologisation traductive. Pour les termes qui ne ressortissent pas à la parole de l'auteur, on n'hésitera pas à faire «éclater» le mot ou l'expression-source en plusieurs traductions-cible différentes (Ladmiral 1979: 223-229).

À la différence de Meschonnic et de Ladmiral qui épousent une approche plutôt prescriptive dans leurs écrits, nous adoptons une approche descriptive. Dans notre corpus la double pratique pour la traduction du lexique comprend trois aspects. Premièrement on traduit les termes techniques de façon imitative par rapport au textesource, c'est-à-dire qu'on procède à des traductions littérales, à des emprunts, ou qu'on utilise des mots apparentés en langue-cible; tandis que pour traduire les termes du lexique général on produit des traductions idiomatiques qui sont souvent nonimitatives. Voici des exemples, tirés des livres Le différend (Lyotard 1983) et The differend (Lyotard 1988), de termes techniques et de leurs traductions: 
1) Traductions littérales

'un tort' - a wrong

'enchaîner' - to link

'enchainement' - linkage

'le monde des noms' - world of names

2) Emprunts (plus naturalisation)

'un différend' - differend

3) Mots apparentés

'genre de discours' - genre of discourse

'régime de phrase' - phrase regimen

'univers de phrase' - phrase universe

'un dommage' - damage

'un litige' - litigation

La traduction imitative peut avoir pour conséquence de produire un lexique plus insolite que celui du texte-source, par exemple differend est un néologisme formel en anglais, alors que 'différend' est un mot existant dans la langue générale en français mais auquel Lyotard attribue un sens spécifique. Le lecteur du texte-cible ne pourra comprendre le sens du terme qu'en lisant les explications contenues dans le texte, alors que le lecteur français aura déjà une idée du sens. La tendance à employer des procédés imitatifs pour la traduction de termes techniques semble si forte que le traducteur est même prêt à lui sacrifier une partie de la clarté du texte. C'est ce qui se produit, par exemple, dans les cas où l'on emploie un mot apparenté en anglais qui a un sens très différent de celui du terme français. Dans le corpus le terme 'instance', qui a chez Lyotard le sens de position et d'autorité, est traduit la plupart du temps par instance qui en anglais veut généralement dire un exemple ou un cas. Voici un deuxième exemple: le terme 'finalité' est souvent traduit par finality; et il est vrai que le dictionnaire $\mathrm{OED}^{2}$ donne pour ce mot le sens de cause finale. Il n'en demeure pas moins cependant que dans son acception la plus courante le terme anglais désigne le caractère de ce qui est définitif. Pour le lecteur anglophone qui ne possède pas de grandes connaissances en philosophie la traduction prête à confusion.

Par rapport à d'autres domaines techniques, la philosophie semble davantage influencée par la forme des termes-source pour traduire les termes techniques. À propos de la terminologie et de sa traduction en général, Reiner Arntz énumère trois procédés traductionnels pour les termes dont il n'y a pas d'équivalents préexistants en langue-cible: emprunts ou traduction littérale; traduction non-littérale; et paraphrase (Arntz 1993: 15-16). C'est à la première catégorie que les traducteurs de la philosophie recourent de préférence.

Le deuxième aspect de la double pratique que l'on trouve dans le corpus pour la traduction du lexique est également mentionné par Meschonnic et par Ladmiral: on maintient la même traduction lors de chaque apparition du terme technique, ce qui n'est pas le cas pour les mots du lexique général. Le troisième aspect est le suivant: on fait usage ou on tient compte tout au moins de traductions antérieures des termes techniques, c'est-à-dire de traductions pré-existantes des termes effectuées par d'autres traducteurs; procédé qui n'entre pas en ligne de compte de façon aussi précise pour ce qui concerne la traduction du lexique général.

Dans les textes de Lyotard apparaissent de nombreuses citations d'autres penseurs, en particulier des Allemands. C'est surtout dans la traduction des termes 
d'autres penseurs que l'on remarque la caractéristique mentionnée ci-dessus, à savoir la reprise de traductions antérieures des termes. La situation est assez complexe, car Lyotard se reporte à la tradition française, tandis que les traducteurs de Lyotard se reportent à la tradition anglaise, de la traduction des penseurs allemands. Dans les deux cas il s'agit de traductions consacrées par l'usage. En voici des exemples:

1) Termes de Kant dans Le différend/The Differend (Lyotard 1983 et 1988)

'le jugement réfléchissant' - reflective judgment

'le schème' - the schema

'les antinomies dynamiques' - dynamical antinomies

2) Termes de Hegel dans «Discussions ou phraser "après Auschwitz" »/«Discussions or phrasing "after Auschwitz" » (Lyotard 1981 et 1989a)

'effectivité' - actuality

'la rationalité positive' - positive reason

3) Termes de Freud dans «Le travail du rêve ne pense pas»/«The dreamwork does not think» (Lyotard 1971 et 1989b)

'les pensées du rêve' - dream-thoughts

'le refoulement' - repression

'la condensation' - condensation

'le déplacement' - displacement

'l'élaboration secondaire' - secondary revision

\section{COMPLEXIFICATION DE LA DISTINCTION : TERMES TECHNIQUES/LEXIQUE GÉNÉRAL DANS LES PROCÉDÉS DE TRADUCTION}

Ayant dégagé les caractéristiques générales des procédés traductionnels du lexique, on se rend compte en réexaminant le corpus qu'il existe de nombreux cas d'exception, et que la situation est en fait plus complexe que celle que nous venons d'esquisser. D'abord en ce qui concerne le lexique général, les traducteurs ont souvent recours aux procédés qui caractérisent le traitement traductionnel des termes techniques. Ils traduisent souvent les mots du lexique général de façon imitative, ce qui produit parfois un langage peu naturel, c'est-à-dire un langage qui ne se conforme pas aux normes d'usage de la langue-cible. Il y a nombre d'exemples où les traducteurs réemploient la même traduction d'un mot qui apparaît plus d'une fois dans le texte, assurant de la sorte la reproduction du tissu cohésif. Quant à l'emploi de traductions antérieures, il s'agit certainement d'un procédé fondamental de la traduction en général.

En ce qui concerne les termes techniques, on constate une certaine complexité au niveau de la réalisation des procédés imitatifs de traduction. Nous aborderons cette question au cours de notre discussion de la non-adoption par les traducteurs des trois procédés caractérisant la traduction des termes techniques. On peut proposer plusieurs raisons à cette non-adoption. Si les traducteurs n'emploient pas la même traduction d'un terme de façon uniforme dans un texte, ou s'ils produisent même parfois une erreur de traduction pour un terme technique, cela peut témoigner d'un travail peu soigné.

Bien plus intéressante est l'idée que les traducteurs ne suivent pas toujours les procédés traductionnels pour les termes techniques parce qu'ils semblent ne pas reconnaître et classer certains mots comme étant des termes techniques. Cette méconnais- 
sance potentielle met en relief le rôle du traducteur/lecteur dans l'attribution du statut de terme technique, ainsi que le rôle du texte traduit comme lecture au sens d'interprétation du texte-source. Ladmiral souligne aussi l'importance et la difficulté de reconnaître les unités terminologiques (Ladmiral 1979: 223). Dans les textes du corpus le non-emploi de traductions antérieures et l'emploi de plusieurs traductionscible semblent indiquer dans la plupart des cas que le traducteur n'a pas reconnu le terme comme étant un terme technique du philosophe. Cela vaut pour les termes d'autres philosophes auxquels Lyotard fait référence, ainsi que pour ses propres termes. Examinons la traduction des termes lyotardiens dans les deux textes traduits (Lyotard 1989c et Lyotard 1992) qui ont comme texte-source "Missive sur l'histoire universelle» (Lyotard 1986). Ce texte inclut un ensemble de termes techniques de Lyotard qui avait déjà été élaboré dans son livre Le différend (Lyotard 1983). Voici les termes ainsi que les traductions-cible:

$\begin{array}{lll}\text { TS } & \text { TT1 } & \mathrm{TT2}^{3} \\ \text { 'le différend' } & \text { disagreement } & \text { differend } \\ \text { 'le litige' } & \text { contention } & \text { litigation } \\ \text { 'genre de discoursive genre } & \text { genre of discourse } \\ \text { 'phrase' } & \text { what follows on from } & \text { phrase } \\ \text { 'enchaînement' } & \text { linkage } \\ \text { 'le monde des noms' } & \text { world of names }\end{array}$

On remarque que le traducteur de la deuxième traduction ${ }^{4}$ emploie les mêmes termes traduits que l'on trouve dans The differend (Lyotard 1988, voir section 3 ci-dessus), c'est-à-dire qu'il reprend des traductions antérieures épousant des procédés traductionnels d'ordre imitatif. Par contre le traducteur de la première traduction ne suit pas ces stratégies qui caractérisent la traduction de termes techniques. On peut supposer que ce traducteur n'a pas reconnu les termes en question. C'est un ensemble de traits du contexte de production de la traduction qui permet de comprendre sa méconnaissance des termes. D'abord le texte-source "Missive» n'est pas un texte qui s'adresse à un public de spécialistes. Les quelques termes techniques de Lyotard n'y sont pas mis en valeur; ils sont enchâssés, presque cachés, dans un texte qui n'a certainement pas l'apparence d'un texte philosophique. Par ailleurs, comme nous l'avons mentionné dans notre discussion sur la terminologie philosophique (voir section 2), les termes lyotardiens ne sont pas des néologismes formels: ils ont une forme et des sens préexistants dans d'autres domaines du langage. Hervey, Higgens et Loughridge parlent du piège que constitue le mot ayant un sens habituel qui ne semble pas hors de propos dans le texte, où ce mot remplit pourtant la fonction de terme technique (Hervey, Higgens et Loughridge 1996: 147). C’est précisément le cas des termes techniques dans "Missive» (et de certains termes techniques d'autres philosophes dans les autres textes du corpus). Hervey, Higgens et Loughridge affirment que dans une telle situation le traducteur peut ne pas identifier le terme comme étant un terme technique, et le traduire incorrectement suivant le sens habituel du mot (Hervey, Higgens et Loughridge 1996: 147).

On pourrait penser qu'une telle éventualité ne surviendra pas si le traducteur possède la connaissance nécessaire du domaine technique en question. Justement, dans le cas qui nous préoccupe, le traducteur de TT1 ne possède pas de connaissances spécialisées de la philosophie lyotardienne, alors que les œuvres de Lyotard jouent un rôle important dans les activités de recherche universitaire du traducteur de $\mathrm{TT}^{5}$. 
Le niveau de connaissances du traducteur dépend au moins en partie du fait que TT1 est un article alors que TT2 est un chapitre dans la traduction d'un livre de Lyotard. Pour la traduction d'un livre le directeur de la publication choisira certainement quelqu'un qui possède de très bonnes connaissances du philosophe à traduire, ce qui ne sera peut-être pas considéré comme essentiel pour la traduction d'un court article. D'une façon générale on investira plus de temps, de recherches, et d'attention aux détails dans la production d'un livre que dans celle d'un article, car un livre, étant beaucoup plus 'visible' qu'un article, peut mettre en cause la réputation de la maison d'édition et du traducteur - ce dernier étant généralement, en l'occurrence, un universitaire prétendant également au rôle de commentateur du philosophe. En préparant sa traduction le traducteur de TT2 de «Missive» a consulté des spécialistes en matière de philosophie et de langue française, tandis que le traducteur de TT1 n'a discuté de son travail qu'avec le directeur de la publication. La collaboration de plusieurs personnes dans un projet de traduction aboutira à un produit plus soigné, car elle permet davantage de discussion d'idées et fournit des moyens de contrôle. Dans le cas de «Missive» il est à noter que TT1 est le fruit du travail d'un seul traducteur, alors que plusieurs personnes ont collaboré pour produire TT2 (voir note 4). Un dernier facteur pertinent dans le contexte de la production est la date de publication des textes: The differend a été publié en 1988, TT1 en 1989, et TT2 en 1992. Une période de quelques années peut être cruciale pour faire connaître les termes d'un penseur contemporain. Le traducteur de TT2 a sans doute bénéficié d'une meilleure connaissance des termes de Lyotard et de leurs traductions antérieures grâce au facteur temporel. On ne peut pas savoir quels facteurs du contexte de production des deux traductions ont réellement été significatifs en l'occurrence, mais la discussion de ce cas indique l'influence potentielle d'éléments du contexte sur le contenu du produit traductionnel en général et plus spécifiquement en ce qui concerne la traduction des termes techniques.

Passons à un autre aspect du facteur temporel qui revêt une importance particulière dans la traduction de textes contemporains. Le traducteur peut ne pas reconnấtre un terme technique du philosophe parce que le concept du philosophe est encore en cours d'élaboration. Nous avons parlé du processus de technicalisation du terme 'différend' (voir section 2). Il est intéressant de constater que, dans une note consacrée à ce terme, la traductrice du texte « Judicieux dans le différend » (Lyotard 1985 et 1989d/1987), le définit selon son acception générale, et ne fait aucune référence aux deux emplois spécifiques du terme dans le texte, 'différend illusoire' et 'différend véritable' (c'est cette distinction qui conduira ultérieurement au sens technique du terme pour Lyotard). La traductrice a choisi d'emprunter le terme 'différend' et de l'employer comme tel dans sa traduction, non pas parce qu'elle a identifié le terme comme un terme technique en voie d'élaboration, mais parce qu'elle n'arrivait pas à trouver un équivalent anglais et que le terme est un terme-clé dans «Judicieux». Le choix de la traductrice a créé un précédent qui a certainement influencé le traitement ultérieur du terme par d'autres traducteurs, notamment le traducteur du livre Le différend (Lyotard 1983). Dans The Differend (Lyotard 1988) ce traducteur écrit également une note à propos du terme: il explique que le terme français est emprunté parce qu'il s'agit d'un terme technique de Lyotard.

La question de l'influence d'un choix effectué par un traducteur sur le choix d'autres traducteurs indique une différence entre deux situations d'élaboration 
terminologique: alors que la terminologie d'un philosophe relève plutôt de l'individualité (voir section 2), la traduction de la terminologie philosophique est une affaire de communauté. L'une des raisons de cette élaboration communautaire est probablement qu'il n'y a pas un seul traducteur qui traduit l'œuvre entière du philosophe, mais un ensemble de traducteurs qui traduisent des textes différents à une même époque. Les commentateurs du philosophe contribuent également à ce qui semble être l'objectif final: l'élaboration de traductions-standard des termes. Avant de parvenir à ce but, il y a parfois des débats et des désaccords. Nous avons constaté que l'une des caractéristiques principales de la traduction de termes philosophiques est l'adoption de procédés traductionnels d'ordre imitatif. L'approche imitative ne détermine pas cependant une unique manière de traduire, d'où la complexification de cette caractéristique: il y a généralement diverses possibilités traductionnelles, et les différents traducteurs ou commentateurs peuvent ne pas tomber d'accord sur le choix d'une traduction pour le terme technique en question. En ce qui concerne les termes lyotardiens un débat a eu lieu à propos de la traduction du terme 'phrase'. Le traducteur de "Discussions ou phraser "après Auschwitz" ", Georges van den Abbeele, (Lyotard 1981 et 1989a/1986) explique dans une longue note les raisons de son choix de l'anglais phrase plutôt que sentence. Il considère que phrase présente l'avantage de pouvoir être utilisé comme substantif et comme verbe (to phrase) sans modification appréciable du sens, et que phrase recouvre des sens qui ne sont pas spécifiquement linguistiques, ce qui convient bien à la notion de Lyotard. Dans sa traduction du livre Le différend (Lyotard 1983 et 1988) van den Abbeele maintient le choix de phrase. Geoffrey Bennington en revanche préfère la traduction sentence. Tout en reconnaissant les avantages de phrase cités par van den Abbeele, il lui préfère sentence pour les raisons suivantes: sentence marque la relation ambivalente entre la philosophie lyotardienne et la tradition philosophique anglo-américaine; l'anglais phrase désigne une partie d'une entité, alors que la 'phrase' de Lyotard constitue une entité; et il est tout aussi étrange de considérer les exemples de Lyotard comme des phrases que comme des sentences (Bennington 1988: 123-124). Ce genre de désaccord ne se résout qu'avec le temps. Phrase semble prévaloir actuellement, probablement du fait que les universitaires anglophones lisent et citent les textes traduits par van den Abbeele.

On pourrait dire que le débat sur la traduction anglaise de 'phrase' a un fondement linguistique en ce sens que les ressources de la langue anglaise offrent deux possibilités de traduction pour un seul terme français. On rencontre la difficulté inverse dans le cas où deux termes techniques en français, souvent conçus comme un couple de termes, ne semblent correspondre qu'à un seul terme en anglais. Tel est le cas dans les textes de Lyotard pour les couples 'pouvoir'/'puissance' et 'savoir'/'connaissance'. Si les traducteurs ne choisissent pas des formes distinctes en anglais pour différencier les termes d'un couple ou ne traduisent pas chaque terme de façon uniforme dans le texte, l'identité des termes techniques se perd. Les différents traducteurs du corpus ont été plus ou moins attentifs à ce problème. Alors que chez certains traducteurs il s'agit de négligence, dans d'autres cas le contexte linguistique ne permet pas toujours de maintenir les termes choisis. Le traducteur de La condition postmoderne (Lyotard 1979 et 1984a) par exemple, explique dans une note qu'en général il traduit 'savoir' par knowledge et 'connaissance' par learning, mais que parfois il était «nécessaire» de traduire cette dernière par knowledge, surtout lorsque 'connaissance' est à la forme du 
pluriel. Le traducteur affirme que le lecteur devrait être à même de discerner le sens voulu d'après le contexte. Il n'en demeure pas moins que l'emploi du même terme pour exprimer deux sens différents ne favorise pas la clarté.

Revenons à la question importante de la standardisation. Il semble que dans certains cas la standardisation du terme traduit ne soit jamais achevée, ou bien qu'elle doive rester provisoire. Cette constatation remet en cause la troisième caractéristique de la traduction des termes techniques que nous avons relevée, la reprise des traductions antérieures. Même pour certains termes des grands philosophes des siècles précédents, il manque des traductions-standard: soit il y a des traductions concurrentes, soit il y a différentes traductions en faveur à des époques différentes. Nous constatons ces traits dans les traductions des textes de Lyotard là où il se réfère aux philosophes allemands, Kant et Hegel. La traductrice de "Judicieux dans le différend» (Lyotard 1985 et 1989d) choisit de traduire les termes hégéliens 'fin' et 'finalité' par purpose et purposiveness (les termes sont en allemand Zweck et Zweckmässigkeit). Le traducteur de Le différend (Lyotard 1983 et 1988) opte par contre pour end et finality pour traduire les mêmes termes, bien qu'il emploie pour les citations le texte Critique of judgement de J. H. Bernard, texte où Bernard utilise les termes purpose et purposiveness. Dans ce cas on est sans doute en droit de parler de traductions concurrentes. Dans le cas de la traduction du terme hégélien aufheben, il s'agit plutôt de phénomènes de mode. Dans l'histoire de la traduction des textes de Hegel tant en français qu'en anglais, il y a eu de nombreuses propositions pour la traduction de ce terme. Les traductions que choisissent Lyotard et le traducteur de «Discussions, ou: phraser “après Auschwitz" ( (Lyotard 1981 et 1989a) reflètent les préférences actuelles pour traduire le terme: 'relever' en français (la traduction de Derrida) et sublate en anglais. L'un des facteurs qui explique l'existence de traductions multiples de certains termes allemands est que le terme allemand recouvre une gamme de sens qui ne correspond pas à un seul terme dans l'autre langue. Tel est le cas pour la traduction du terme Geist en anglais. Le traducteur de «Auschwitz» (Lyotard 1981 et 1989a) choisit de traduire ce terme par mind de façon uniforme dans le texte (probablement parce qu'il utilise le texte The phenomenology of mind de J.B. Baillie pour les citations), bien qu'à certains moments spirit eût été plus approprié.

Pour traduire les termes techniques de penseurs allemands, les traducteurs de Lyotard n'ont pas toujours recours aux traductions-standard en anglais, là où ils subissent l'influence de la traduction française des termes allemands. On en trouve des exemples dans la traduction de "Le travail du rêve ne pense pas» (Lyotard 1971 et 1989b) : pour traduire Rücksicht auf Darstellbarkeit, au lieu d'employer la traductionstandard du terme freudien, considerations of representability, la traductrice emploie l'expression considerations of figurability, qui imite la traduction française, 'prise en considération de la figurabilité. La raison pour laquelle la traductrice est influencée par la forme française est probablement que la notion de 'figure'/figure est centrale dans le texte de Lyotard. Dans ce cas l'influence de la forme française indique que pour la traductrice c'est le texte de Lyotard qui prend le pas sur les textes enchâssés d'autres penseurs et sur les traductions-standard des termes de ces penseurs.

Avant de terminer nos remarques sur la complexification de la distinction entre les procédés traductionnels pour le lexique, il convient de mentionner la pratique du transfert de termes du texte-source au texte-cible, qui est une spécificité importante de la traduction du lexique dans les textes philosophiques. Comme cette pratique 
concerne à la fois les termes techniques et le lexique général, elle refuse la distinction que nous avons dressée entre le traitement des deux types de mots. Les mots étrangers apparaissant dans le texte-source (la plupart du temps des termes techniques ou des termes-clé en allemand, en latin ou en grec) sont reproduits dans la traduction. Ce qui frappe beaucoup plus, c'est le transfert fréquent de mots français aux textes traduits. Généralement le mot ou le syntagme français est mis en italiques et entre parenthèses après sa traduction anglaise. Dans certains cas il y a transfert d'un mot étranger et de son équivalent français au texte anglais, ce qui produit un texte trilingue, comme par exemple dans le cas suivant du traitement d'un terme hégélien dans la traduction de "Auschwitz» (Lyotard 1981 et 1989a) : near itself (an sich) ('auprèsde-soi'). Il semble y avoir toute une gamme de raisons pour le transfert des mots français, dont voici quelques-unes : le mot est un terme technique ou un terme-clé de Lyotard ou d'autres philosophes; un jeu de mots français est perdu dans la traduction anglaise; il y a difficulté à reproduire des distinctions lexicales (par exemple 'langue'/'langage'); le traducteur a choisi différentes traductions-cible pour un seul mot français dans différents endroits du texte traduit; et le terme français est insolite. On pourrait poser comme hypothèse que la présence de mots étrangers pour des termes techniques (ou des termes-clé) dans les textes philosophiques au départ a donné lieu à la pratique du transfert de termes techniques dans les textes traduits, et que cette pratique a été ensuite généralisée à d'autres fonctions concernant le lexique général.

\section{LE TRAITEMENT DU LEXIQUE PHILOSOPHIQUE: STATUT ET EXPLICATIONS}

La complexité du traitement du lexique philosophique ne vient pas contredire l'existence des caractéristiques générales de la traduction des termes techniques et du lexique général. Quel est donc le statut de ces caractéristiques générales? S’agit-il seulement d'une régularité descriptive? L'examen de deux traductions d'un même texte-source par le même traducteur nous aidera peut-être à trouver une réponse à ces questions. Van den Abbeele a traduit un extrait du livre Le différend (Lyotard 1983), publié comme article de revue (Lyotard 1984b) qu'il a ensuite utilisé comme 'brouillon' en effectuant la traduction intégrale du livre (Lyotard 1988). Le tableau suivant montre les termes techniques du texte-source qui ont été traduits différemment dans les deux textes traduits (TT1 est la traduction de 1984b, et TT2 celle de 1988):

$\begin{array}{lll}\text { TS } & \text { TT1 } & \text { TT2 } \\ \text { 'tort' } & \text { injustice } & \text { wrong } \\ \text { 'dommage' } & \text { injury } & \text { damage } \\ \text { 'il arrive' } & \text { it occurs/happens } & \text { it happens } \\ \text { 'actuel' } & \text { actual/in question } & \text { current } \\ \text { 'connaissance' } & \text { knowledge } & \text { cognition } \\ \text { 'sens' } & \text { meaning } & \text { sense }\end{array}$

Pour traduire 'tort' et 'dommage' le traducteur emploie des procédés imitatifs dans TT2, traduction littérale et un mot apparenté, alors que ces procédés ne sont pas adoptés dans TT1. Dans TT2 pour traduire 'il arrive' et 'actuel' le traducteur emploie de façon uniforme une seule traduction-cible pour chaque terme, alors que dans 
TT1 il y a plusieurs traductions-cible. Dans TT2 le traducteur traduit 'connaissance' par cognition pour la distinguer de 'savoir'/knowledge (voir la discussion de la section précédente); quant à TT1, la distinction entre 'connaissance' et 'savoir' n'est pas maintenue dans la traduction. Dans TT2 le traducteur choisit sense pour traduire 'sens' parce qu'il s'agit d'un terme de Frege que l'on a traduit précédemment de cette façon; dans TT1 le traducteur ne semble pas reconnaître ce mot comme étant un terme technique de Frege. Quant au lexique général on constate que l'expression de TT2 est plus idiomatique que celle de TT1 grâce à l'emploi d'un registre approprié et au recours à des procédés traductionnels non-imitatifs. Dans TT1 par contre on trouve des formulations étranges, c'est-à-dire peu conformes aux usages de l'anglais, là où l'expression en texte-source n'est ni étrange ni insolite.

En examinant d'autres différences entre TT1 et TT2 on constate que pour produire TT2 le traducteur a modifié TT1 de manière à améliorer le premier texte: par exemple, dans TT2 le traducteur a reproduit le sens de TS de façon plus claire, et il a corrigé des erreurs de traduction de TT1. On peut penser que le traducteur a modifié TT1 en suivant des conceptions d'idéal pour ce genre de traduction. Les procédés qu'il a épousés dans TT2 pour traduire les termes techniques et le lexique général représenteraient donc des procédés idéaux. Les réponses aux questionnaires envoyés aux traducteurs du corpus (voir note 5) permettent de savoir s'il s'agit là d'idéaux personnels ou d'idéaux partagés par d'autres traducteurs (que certains chercheurs nomment des 'normes traductionnelles'). Toutes les personnes interrogées s'accordent pour distinguer le traitement du lexique général et celui du lexique technique. Pour le lexique général les traducteurs préfèrent la traduction idiomatique, tandis qu'ils reconnaissent l'emploi de procédés imitatifs, l'importance de l'uniformité de choix, et le rôle de traductions antérieures dans la traduction du lexique technique. Cependant les déclarations des traducteurs en ce qui concerne l'emploi de procédés imitatifs sont nuancées par rapport aux données que fournissent les textes traduits eux-mêmes, où la pratique imitative est très marquée. On pourrait poser comme hypothèse que les procédés traductionnels du lexique philosophique constituent des idéaux qui conditionnent la production traductive, mais dont les traducteurs ne sont pas pleinement conscients.

Nous venons d'élaborer la notion d'idéaux normatifs gouvernant la double pratique dans la traduction du lexique philosophique; cette notion ne nous aide pas cependant à comprendre à un niveau plus fondamental pourquoi la double pratique existe. Pour ce faire, il faut chercher ailleurs des éclaircissements. Nous nous pencherons surtout sur la question des termes techniques. L'emploi de procédés traductionnels d'ordre imitatif pour les termes principaux dans les textes philosophiques résulte pour Meschonnic d' "une confusion entre le concept et le mot» (Meschonnic 1985 : 219), confusion qui consiste à penser que si l'on s'éloigne de la forme du mot, on changera le sens. Ladmiral parle d'une métaphysique substantialiste du langage: « comme si la possibilité d'un transcodage des signifiants-source aux signifiants-cible était garantie par la permanence quasi ontologique d'on ne sait quels atomes de signifié» (Ladmiral 1979: 67). Ce mode de pensée pourrait provenir d'une certaine aspiration à l'universalité qui serait propre au discours philosophique, discours qui est une "coïncidence de la singularité individuelle et de l'universalité » (Ladmiral 1983 : 254), qui «joue à être dans le langage, dans tout le langage, alors qu'il ne peut se dérouler que dans une langue» (Rey 1990: 777). On pourrait rapprocher cette 
attitude de celle d'autres disciplines qui aspirent à l'internationalisme (Pinchuck 1977 : 185), où l'on privilégie des dénominations de concepts présentant une grande similitude de signifiants d'une langue à l'autre (Gaudin 1995: 235). Ces modes de pensée influencent sûrement la pratique du traducteur, mais on peut proposer une source d'influence qui est plus immédiate et plus forte: l'attente des lecteurs des textes traduits.

Le traducteur (ainsi que le directeur de publications) est sensible aux préférences et aux besoins des lecteurs, qui influencent donc les pratiques traductionnelles. Basées sur les remarques d'autres chercheurs et sur notre enquête auprès d'un groupe de lecteurs ${ }^{6}$, nous proposons les réflexions suivantes qui mettent en avant la primauté des désirs des lecteurs et de la fonction que remplissent pour eux les textes et les termes traduits. Les lecteurs veulent avant tout comprendre les idées du philosophe. Ils veulent aussi que les traductions rendent le style et l'idiolecte du philosophe cette attente tient au moins en partie à l'attitude respectueuse du public face aux textes de philosophie française qui jouissent d'un prestige particulier dans les milieux universitaires anglophones. La traduction idiomatique du lexique général permet aux lecteurs de comprendre la partie du texte que constituent ces mots; alors que la traduction imitative permet de saisir les spécificités de l'idiolecte du philosophe, y compris ses termes techniques. Les lecteurs vont réutiliser les termes techniques et les termes-clés du philosophe dans leur propre discours, donc ils ont besoin de traductions-cible qui sont succinctes et reconnaissables. D'abord un terme doit être traduit par un terme - une paraphrase explicative ne fera pas l'affaire. Ensuite on pourrait avancer que le traducteur marque d'un «index terminologique» (Ladmiral 1979: 229) les termes centraux, pour permettre aux lecteurs de les reconnaître. L'emprunt d'un terme étranger, l'emploi d'un néologisme ou d'une expression étrange ou inconnue en langue-cible résultant d'une traduction littérale, et le recours à un mot apparenté en langue-cible qui n'est pas employé au sens habituel, constituent autant d'index terminologiques qui indiquent au lecteur qu'il s'agit d'un concept inexistant dans la culture-cible jusque-là, c'est-à-dire d'un terme particulier du philosophe. Les procédés imitatifs servent ainsi à fournir au lecteur des formes distinctes et distinctives. Il est à remarquer que là où le traducteur emploie des expressions inconnues ou étranges, ou des sens inhabituels d'une forme connue, le lecteur apprend le sens voulu en lisant le texte: autrement dit, le texte lui-même fait émerger le contenu sémantique (Ladmiral 1979: 228), ou bien l'explication des termes dans des paratextes (notes, glossaires) rédigés par le traducteur assure la compréhension du lecteur. Les nouveaux mots ou les nouveaux sens de mots préexistants servent ensuite à enrichir la langue-cible.

La permanence d'une même forme du signifiant est également un index terminologique, car c'est le propre d'un terme que de maintenir son unité spécifique faire éclater l'unité spécifique du mot-source le priverait de la fonction terminologique (Ladmiral 1979: 220). Dans un seul texte traduit l'uniformité de traduction d'un terme traduit est essentielle pour que le lecteur suive le texte, car les termes techniques constituent des balises autour desquelles le lecteur construit le sens du texte. La prise en compte de traductions antérieures des termes techniques indique l'importance des liens intertextuels dans le but éventuel d'une standardisation des termes traduits. Même si la stabilisation des termes traduits n'est pas toujours permanente, la standardisation (au moins provisoire) est nécessaire pour assurer la 
compréhension de la part des lecteurs d'un ensemble de textes. Que ce soit dans un seul texte ou dans un ensemble de textes, c'est la cohérence terminologique qui garantit la «consistance» théorique; elle doit donc être conservée en traduction (Rey 1990 : 779). La standardisation des termes traduits assure également l'efficacité de la communication et donc la dissémination en culture-cible de la pensée du philosophe grâce au réemploi des termes par les lecteurs; la reproduction de la «marque théorique» du philosophe (Lamont 1987: 592), grâce aux traductions-cible distinctives qui sont employées de façon uniforme, constitue une condition pour que les termes soient mis en circulation et que les ouvres du philosophe soient légitimées dans la culture-cible. Nous attribuons donc aux lecteurs un rôle primordial non seulement en ce qui concerne les pratiques traductionnelles, mais aussi en ce qui concerne la 'vie' du texte traduit et des termes techniques en traduction. Mais, bien entendu, les lecteurs ne sont pas les seuls à employer les termes techniques. Au cours d'échanges, de négociations et de conflits à travers les circuits d'édition, les colloques, etc., traducteurs, éditeurs, directeurs de publications, lecteurs, et commentateurs contribuent à l'élaboration des termes techniques en traduction, à leur mise en circulation, et à la stabilisation des termes-standard. Au-delà des termes individuels, la mise en place de textes traduits-standard constitue également un objectif important de la communauté impliquée dans la traduction et dans l'utilisation de textes philosophiques.

\section{CONCLUSION}

L'étude descriptive de la traduction des termes philosophiques dans un corpus nous a permis dans un premier temps d'en dégager les caractéristiques principales. Nous avons discuté ensuite de la complexité de la traduction du lexique, qui est due à un certain nombre de facteurs comprenant des questions linguistiques et textuelles, des aspects du contexte de la production des traductions, et le rôle de l'histoire et d'une communauté de traducteurs et d'autres personnes dans l'élaboration des termes traduits. Pour expliquer les spécificités de la traduction du lexique philosophique et surtout le traitement des termes techniques, il est également nécessaire de considérer le contexte social, en particulier le rôle des lecteurs. Une approche contextuelle et sociologique s'avère donc nécessaire pour comprendre l'élaboration et le fonctionnement des termes, non seulement dans l'étude de la terminologie (voir Boulanger 1995), mais aussi dans l'étude de la traduction de la terminologie ${ }^{7}$.

\section{NOTES}

1. Nous faisons la distinction suivante entre 'mots-clés' et 'termes techniques': on trouve les mots-clés dans un seul texte, alors que l'on retrouve les termes techniques dans d'autres textes du philosophe.

2. Oxford English Dictionary

3. $\mathrm{TS}=$ Texte-source

TT1 = Texte traduit 1

TT2 = Texte traduit 2

4. TT2 fait partie d'un livre qui constitue un travail collectif d'un groupe de traducteurs. Une personne a cependant assuré la direction de la publication et la co-traduction de tous les textes traduits; pour les besoins de cet article nous appelons cette personne le traducteur de TT2.

5. Dans le cadre de nos recherches nous avons envoyé des questionnaires aux traducteurs des textes du corpus, lesquels ont fourni des informations sur le contexte de production des traductions, et sur leurs préférences en matière de procédés traductionnels pour les œuvres philosophiques contemporaines. 
6. Dans le cadre de nos recherches nous avons interrogé des professeurs et des étudiants australiens qui lisent les traductions anglaises d'œuvres de philosophie française contemporaine.

7. L'auteur remercie chaleureusement Annick Bouchet et James Wheatley pour leur lecture du présent article.

\section{RÉFÉRENCES}

Arntz, R. (1993): «Terminological equivalence and translation», in Terminology: Applications in Interdisciplinary Communication. Helmi Sonneveld \& Kurt Loening (éds), Amsterdam, John Benjamins.

Bennington, G. (1988) : Lyotard: Writing the Event. Manchester, Manchester University Press.

BoulAnger, J.-C. (1995): «Présentation: Images et parcours de la socioterminologie», Meta 402, pp. 194-205.

Gaudin, F. (1995): «Champs, clôtures et domaines: Des langues de spécialités à la culture scientifique», Meta 40-2, pp. 229-237.

Hervey, S., I. Higgens et M. Loughridge (1996) : «Technical translation», in Thinking German Translation; A Course in Translation Method, German to English. London, New York, Routledge.

Ladmiral, J.-R (1979): Traduire: Théorèmes pour la traduction. Paris, Petite Bibliothèque Payot.

— (1983): «La traduction philosophique», Revue de phonétique appliquée 66-68, pp. 233-259.

Lamont, M. (1987): «How to become a dominant French philosopher: The case of Jacques Derrida ", American Journal of Sociology 93-2, pp. 584-622.

Meschonnic, H. (1985) : «Poétique d'un texte de philosophe et de ses traductions », in Les tours de Babel. France, Éditions Trans-Europ-Repress.

Peтioky, V. (1994) : «Zur Übertragung philosophischer Texte; Am Beispiel von Übertragungen Deutsch-Russisch ", in Translation Studies - An Interdiscipline. Mary SNell-Hornby, Franz PöchHacker, \& Klaus KaIndl (éds), Amsterdam/Philadelphia, John Benjamins.

Pinchuck, I. (1977): Scientific and Technical Translation. London, Andre Deutsch.

Rey, A. (1990) : «Lexico-logiques, discours, lexiques et terminologies "philosophiques" », in Encyclopédie philosophique universelle, tome 2, Les notions, Paris, PUF.

Roth-Neves, R. (2000): "On justification in Translation Studies: Some comments and a research report», Target 12-1, pp. 1-29.

\section{TEXTES DU CORPUS}

Lyotard, J.-F. (1971): «Le travail du rêve ne pense pas», in Discours, figure. Paris, Éditions Klincksieck.

- (1979): La condition postmoderne: Rapport sur le savoir. Paris, Éditions de Minuit.

— (1981): "Discussions, ou: phraser "après Auschwitz" ", in Les fins de l'homme: à partir du travail de Jacques Derrida. P. Lacoue-Labarthe et J-L. Nancy (éds). Paris, Éditions Galilée.

- (1983): Le différend. Paris, Les Éditions de Minuit.

- (1984a): The Postmodern Condition: A Report on Knowledge. Traduit par Geoffrey Bennington et Brian Massumi. Manchester, Manchester University Press.

— (1984b): "The différend, the referent, and the proper name», Traduit par Georges van den Abbeele. Diacritics fall 1984: 4-14.

— (1985): «Judicieux dans le différend», in La faculté de juger. Lyotard et al. Paris, Éditions de Minuit.

— (1986): «Missive sur l'histoire universelle», in Le postmoderne expliqué aux enfants. Paris, Éditions de Minuit.

— (1988): The Differend: Phrases in Dispute. Traduit par Georges van den Abbeele. Minneapolis, University of Minnesota Press.

—— (1989a): "Discussions, or phrasing "after Auschwitz" ", Traduit par Georges van den Abbeele, in The Lyotard Reader. Andrew Benjamin (éd). Oxford/Cambridge, Massachusetts: Basil Blackwell. (première parution du texte en 1986) 
- (1989b): «The dream-work does not think», Traduit par Mary Lydon, in The Lyotard Reader. Andrew Benjamin (éd). Oxford/Cambridge, Massachusetts, Basil Blackwell.

- (1989c) : "Universal history and cultural differences», Traduit par David Macey, in The Lyotard Reader. Andrew Benjamin (éd). Oxford/Cambridge, Massachusetts, Basil Blackwell.

— (1989d): «Judiciousness in dispute, or Kant after Marx», Traduit par Cecile Lindsay, in The Lyotard Reader. Andrew Benjamin (éd). Oxford/Cambridge, Massachusetts: Basil Blackwell. (première parution du texte en 1987)

— (1992): «Missive on universal history», in The Postmodern Explained to Children. Julian Pefanis and Morgan Thomas (éds). Sydney, Power Publications. 